\title{
INEQUALITIES FOR THE TRACE OF MATRIX EXPONENTIALS*
}

\section{DENNIS S. BERNSTEIN†}

Abstract. Several inequalities involving the trace of matrix exponentials are derived. The Golden-Thompson inequality $\operatorname{tr} e^{A+B} \leqq \operatorname{tr} e^{A} e^{B}$ for symmetric $A$ and $B$ is obtained as a special case along with the new inequality $\operatorname{tr} e^{A} e^{A^{T}} \leqq \operatorname{tr} e^{A+A^{T}}$ for nonnormal $A$.

Key words. matrix exponential, inequality, trace

AMS(MOS) subject classification. 15

1. Introduction. For $n \times n$ real symmetric matrices $A$ and $B$, the Golden-Thompson inequality [1]-[5] states that

$$
\operatorname{tr} e^{A+B} \leqq \operatorname{tr} e^{A} e^{B}
$$

Reference [5] generalizes (1.1) to allow arbitrary spectral functions in place of the trace operator and provides an overview of applications in which these inequalities arise.

In contrast to (1.1), problems in linear-quadratic optimal feedback control [6] typically involve a performance functional $J$ of the form

$$
J=\operatorname{tr} \int_{0}^{\infty} e^{A t} V e^{A^{T} t} R d t
$$

where $V$ and $R$ denote noise intensity and performance weighting matrices, respectively, and $A$ denotes the linear system dynamics matrix. The form of (1.2) thus suggests inequalities of the form (1.1) involving $A$ and $A^{T}$, where $A$ is nonnormal, in place of symmetric $A$ and $B$. Such inequalities are motivated by robust sampled-data controldesign problems which require performance bounds for uncertain system models.

The main result of the present note is the inequality

$$
\operatorname{tr} e^{A} e^{A^{T}} \leqq \operatorname{tr} e^{A+A^{T}}
$$

Rather surprisingly, the sign of the inequality (1.3) is opposite to the sign of (1.1). To understand why this is the case, we derive a series of inequalities which, upon appropriate specialization, yield both (1.1) and (1.3).

2. Inequalities. The following lemma is required. (Let $C^{T}$ denote the transpose of a matrix $C$.)

LEMMA 2.1. If $C \in R^{n \times n}$ and $r$ is a positive integer, then

$$
\operatorname{tr} C^{2 r} \leqq \operatorname{tr} C^{r} C^{r T} \leqq \operatorname{tr}\left(C C^{T}\right)^{r} .
$$

Proof. The first inequality follows from $\operatorname{tr}\left(C^{r}-C^{r T}\right)\left(C^{r T}-C^{r}\right) \geqq 0$, while the second follows from a result of $\mathrm{K}$. Fan (see [4, pp. 234, 516]).

THEOREM 2.1. If $A, B \in R^{n \times n}$, then

$$
\begin{aligned}
& \operatorname{tr} e^{A+B} \leqq \operatorname{tr} e^{(A+B) / 2} e^{(A+B)^{T} / 2} \leqq \operatorname{tr} e^{\left(A+A^{T}+B+B^{T}\right) / 2} \\
& \leqq \operatorname{tr} e^{\left(A+A^{T}\right) / 2} e^{\left(B+B^{T}\right) / 2} \leqq \frac{1}{2} \operatorname{tr}\left(e^{A+A^{T}}+e^{B+B^{T}}\right), \\
& \left.\begin{array}{l}
\operatorname{tr} e^{A} e^{B} \\
\frac{1}{2} \operatorname{tr}\left(e^{2 A}+e^{2 B}\right)
\end{array}\right\} \leqq \frac{1}{2} \operatorname{tr}\left(e^{A} e^{A^{T}}+e^{B} e^{B^{T}}\right) \leqq \frac{1}{2} \operatorname{tr}\left(e^{A+A^{T}}+e^{B+B^{T}}\right) .
\end{aligned}
$$

* Received by the editors January 21, 1987; accepted for publication May 11, 1987. This research was supported in part by the Air Force Office of Scientific Research under contract F49620-86-C-0002.

$\dagger$ Harris Corporation, Melbourne, Florida 32902. 
Proof. Defining $C=e^{A / 2 r} e^{B / 2 r}$, (2.1) becomes

$$
\operatorname{tr}\left(e^{A / 2 r} e^{B / 2 r}\right)^{2 r} \leqq \operatorname{tr}\left(e^{A / 2 r} e^{B / 2 r}\right)^{r}\left(e^{B^{T} / 2 r} e^{A^{T} / 2 r}\right)^{r} \leqq \operatorname{tr}\left(e^{A / 2 r} e^{B / 2 r} e^{B^{T} / 2 r} e^{A^{T} / 2 r}\right)^{r} .
$$

Letting $r \rightarrow \infty$, the exponential product formula [5, p. 60] and its generalization [7, p. 97] yield the first two inequalities of (2.2). The third inequality of (2.2) follows from Corollary 3 of [5] while the fourth inequality of (2.2) follows from

$$
0 \leqq \operatorname{tr}\left[e^{\left(A+A^{T}\right) / 2}-e^{\left(B+B^{T}\right) / 2}\right]^{2} .
$$

To prove (2.3) note that the upper leftmost inequality follows from $0 \leqq$ $\operatorname{tr}\left(e^{A}-e^{B}\right)\left(e^{A}-e^{B}\right)^{T}$. The remaining inequalities in (2.3) follow from $\operatorname{tr} e^{2 A} \leqq$ $\operatorname{tr} e^{A} e^{A^{T}} \leqq \operatorname{tr} e^{A+A^{T}}$, which is a consequence of (2.2) with $B=A$.

COROLLARY 2.1. If $A \in R^{n \times n}$, then

$$
\begin{aligned}
& \operatorname{tr} e^{2 A} \leqq \operatorname{tr} e^{A} e^{A^{T}} \leqq \operatorname{tr} e^{A+A^{T}} \leqq \frac{n}{2}+\frac{1}{2} \operatorname{tr} e^{2\left(A+A^{T}\right)}, \\
& \operatorname{tr} e^{2 A} \leqq \frac{n}{2}+\frac{1}{2} \operatorname{tr} e^{2 A} e^{2 A^{T}} \leqq \frac{n}{2}+\frac{1}{2} \operatorname{tr} e^{2\left(A+A^{T}\right)} .
\end{aligned}
$$

If $A, B \in R^{n \times n}$ are symmetric, then

$$
\operatorname{tr} e^{A+B} \leqq \operatorname{tr} e^{A} e^{B} \leqq \frac{1}{2} \operatorname{tr}\left(e^{2 A}+e^{2 B}\right) .
$$

Proof. The first two inequalities of (2.4) follow from the first two inequalities of (2.2) with $B=A$. The last inequality of (2.4) follows from the last inequality of (2.2) with $B=0$ and $A$ replaced by $2 A$. Inequalities (2.5) follow from (2.3) with $B=0$ and $A$ replaced by $2 A$ while ignoring the lower leftmost term in (2.3). Finally, (2.6) follows from (2.2).

Remark. The second inequality in (2.4) and the first inequality in (2.6) correspond to (1.3) and (1.1), respectively.

3. Additional inequalities. The question immediately arises as to whether any additional inequalities involving the expressions appearing in (2.4) and (2.5) are true. Note that $\operatorname{tr} e^{A} e^{B}$ in (2.3) cannot be merged with (2.2) because of the sign reversal between (1.1) and (1.3). It can readily be seen that the only remaining possibilities are

$$
\begin{aligned}
& \operatorname{tr} e^{\left(A+A^{T}\right) / 2} e^{\left(B+B^{T}\right) / 2} \stackrel{\frac{1}{2}}{2} \operatorname{tr}\left(e^{A} e^{A^{T}}+e^{B} e^{B^{T}}\right), \\
& \operatorname{tr} e^{\left(A+A^{T}\right) / 2} e^{\left(B+B^{T}\right) / 2} \stackrel{?}{\frac{1}{2}} \operatorname{tr}\left(e^{2 A}+e^{2 B}\right), \\
& \operatorname{tr} e^{A} e^{B} \stackrel{?}{\frac{1}{2}} \operatorname{tr}\left(e^{2 A}+e^{2 B}\right) .
\end{aligned}
$$

By randomly generating $A$ and $B,(3.1)$ was shown to be false. Since (3.2) implies (3.1), (3.2) must also be false. Furthermore, in the case $B^{T}=-B$, inequality (3.1), which becomes

$$
\operatorname{tr} e^{\left(A+A^{T}\right) / 2} \stackrel{?}{\underline{?}} \frac{n}{2}+\frac{1}{2} \operatorname{tr} e^{A} e^{A^{T}},
$$

was also shown to be false. Hence (2.4) and (2.5) cannot be merged. Finally, the remaining inequality (3.3) was also shown to be false even when $B=0$.

Remark. The results of this paper can be generalized to the case in which $A$ and $B$ are complex matrices. Generalization to arbitrary spectral functions [5] remains an area for further research. 
Acknowledgment. I wish to thank Scott W. Greeley for carrying out numerical calculations which suggested the results of this paper.

\section{REFERENCES}

[1] S. GolDEN, Lower bounds for the Helmholtz function, Phys. Rev., 137 (1965), pp. B1127-B1128.

[2] C. J. THOMPson, Inequality with applications in statistical mechanics, J. Math. Phys., 6 (1965), pp. 18121813.

[3] A. LENARD, Generalization of the Golden-Thompson Inequality $\operatorname{Tr}\left(e^{A} e^{B}\right) \geqq \operatorname{Tr} e^{A+B}$, Indiana Univ. Math. J., 21 (1971), pp. 457-467.

[4] A. W. MARSHALl AND I. OlKIN, Inequalities: Theory of Majorization and Its Applications, Academic Press, New York, 1979.

[5] J. E. Cohen, S. Friedland, T. KATO, AND F. P. Kelly, Eigenvalue inequalities for products of matrix exponentials, Linear Algebra Appl., 45 (1982), pp. 55-95.

[6] K. KwakeRnaAK AND R. Sivan, Linear Optimal Control Systems, John Wiley, New York, 1972.

[7] V. S. VARadarajan, Lie Groups, Lie Algebras, and Their Representations, Springer-Verlag, Berlin, New York, Heidelberg, 1984. 